\title{
Consistent Fuzzy Linguistic Preference Relations for Computer Integrated Manufactory Systems Selection
}

\author{
Tien-Chin Wang ${ }^{1}$ Yueh-Hsiang Chen ${ }^{2}$ \\ ${ }^{1}$ Department of Information Management, I-Shou University, Kaohsiung, 840, Taiwan \\ ${ }^{2}$ Doctoral Student, Department of Information Engineering, I-Shou University, Kaohsiung 840, Taiwan; Lecturer, \\ Department of Information Management, Kao Yuan University, Kaohsiung 821, Taiwan
}

\begin{abstract}
Most decision-making and problem-solving tasks are too complex to be understood quantitatively. People perform such tasks using knowledge that is imprecise rather than precise. This study presents consistent fuzzy linguistic preference relations rather than fuzzy analytic hierarchy process (FAHP) to select computer integrated manufactory (CIM) systems. The proposed method yields decision matrices for making pairwise comparisons using additive transitivity. Only $n-1$ comparison judgments are required to ensure consistency on a level that contains $n$ criteria.
\end{abstract}

Keywords: consistent fuzzy linguistic preference relations; FAHP; CIM; consistency

\section{Introduction}

Zadeh [1] introduced the fuzzy set theory in 1965, to rationalize uncertainty associated with impression or vagueness, and thus applicable to human thought. The AHP method can be used to express experts' opinions, but cannot model human thinking so fuzzy AHP, a fuzzy extension of AHP [2], was developed to solve hierarchical imprecise problems.

Consistency is crucial for achieving correct solutions in decision process. Due to each positive reciprocal matrix is described by fuzzy numbers in FAHP, so satisfying consistency is very difficult. Furthermore, establishing a fuzzy positive reciprocal matrix requires $n \times(n-1)$ judgments to be made for a level with $n$ criteria. The numbers of comparisons increases with the number of criteria, so inconsistent conditions are likely to occur.

In order to solve the consistency problem, we adopt consistent fuzzy linguistic preference relations to construct fuzzy decision matrix instead of fuzzy positive reciprocal matrix. The proposed method yields decision matrices for making pairwise comparisons using additive transitivity. Only $n-1$ comparison judgments are required to ensure consistency on a level that contains $n$ criteria. Finally, the proposed method is demonstrated by applying it to select CIM systems [3].

\section{Consistent fuzzy linguistic preference relations}

The consistent fuzzy linguistic preference relations method was proposed by Wang and Chen [4] to deal with vagueness judgments. This study presents the proposed method to extend on consistency of Fuzzy AHP. The proposed method constructs fuzzy preference relations matrices using fuzzy linguistic assessment variables $\widetilde{P}=\left(\widetilde{p}_{i j}\right)=\left(p_{i j}^{L}, p_{i j}^{M}, p_{i j}^{R}\right)$ based on consistent fuzzy preference relations [5]. Table 1 lists the fuzzy linguistic assessment variables.

Table 1. Fuzzy linguistic assessment variables

\begin{tabular}{ll} 
Linguistic variables & Fuzzy numbers \\
\hline Very Poor (VP) & $\left(0, p_{V P}^{M}, p_{V P}^{R}\right)$ \\
$\vdots$ & $\vdots$ \\
Medium (M) & $\left(p_{M}^{L}, 0.5, p_{M}^{R}\right)$ \\
$\vdots$ & $\vdots$ \\
Very Good (VG) & $\left(p_{V G}^{L}, p_{V G}^{M}, 1\right)$ \\
\hline
\end{tabular}

Buckley [6] defines the consistency of a fuzzy positive reciprocal matrix as follows.

Definition 2.1. A fuzzy positive matrix $\widetilde{A}=\left(\widetilde{a}_{i j}\right)$ is reciprocal if and only if $\widetilde{a}_{i j} \otimes \widetilde{a}_{j i} \approx \widetilde{1}$, where $\tilde{1}=(1,1,1)$.

Definition 2.2. A fuzzy positive reciprocal matrix $\widetilde{A}=\left(\widetilde{a}_{i j}\right)$ is consistent if and only if $\widetilde{a}_{i j} \otimes \widetilde{a}_{j k} \approx \widetilde{a}_{i k}$.

Wang and Chen [4] proposed consistent fuzzy linguistic preference relations. These propositions are described as follows.

Proposition 2.1. Suppose a set of alternatives, $X=\left\{x_{1}, \ldots, x_{n}\right\}$ associated with a fuzzy reciprocal multiplicative preference matrix $\widetilde{A}=\left(\widetilde{a}_{i j}\right)$ with $\widetilde{a}_{i j} \in[1 / 9,9]$, then the corresponding fuzzy 
reciprocal linguistic preference relation, $\widetilde{P}=\left(\widetilde{p}_{i j}\right)=\frac{1}{2}\left(1+\log _{9} \tilde{a}_{i j}\right)$ with $\quad \widetilde{p}_{i j} \in[0,1]$, verifies the additive reciprocal, namely, the following statements are equivalent.

(1) $p_{i j}^{L}+p_{j i}^{R} \approx 1 \quad \forall i, j \in\{1, \ldots, n\}$.

(2) $p_{i j}^{M}+p_{j i}^{M} \approx 1 \quad \forall i, j \in\{1, \ldots, n\}$.

(3) $p_{i j}^{R}+p_{j i}^{L} \approx 1 \quad \forall i, j \in\{1, \ldots, n\}$.

Proposition 2.2. For a reciprocal fuzzy linguistic preference relation $\widetilde{P}=\left(\tilde{p}_{i j}\right)=\left(p_{i j}^{L}, p_{i j}^{M}, p_{i j}^{R}\right)$ to be consistent, the following statements must be equivalent:

(1) $p_{i j}^{L}+p_{j k}^{L}+p_{k i}^{R} \approx \frac{3}{2}, \forall i<j<k$

(2) $p_{i j}^{M}+p_{j k}^{M}+p_{k i}^{M} \approx \frac{3}{2}, \forall i<j<k$

(3) $p_{i j}^{R}+p_{j k}^{R}+p_{k i}^{L} \approx \frac{3}{2}, \forall i<j<k$

(4) $p_{i(i+1)}^{L}+p_{(i+1)(i+2)}^{L}+\ldots+p_{(j-1) j}^{L}+p_{j i}^{R} \approx \frac{j-i+1}{2}, \forall i<j$

(5) $p_{i(i+1)}^{M}+p_{(i+1)(i+2)}^{M}+\ldots+p_{(j-1) j}^{M}+p_{j i}^{M} \approx \frac{j-i+1}{2}, \forall i<j$

(6) $p_{i(i+1)}^{R}+p_{(i+1)(i+2)}^{R}+\ldots+p_{(j-1) j}^{R}+p_{j i}^{L} \approx \frac{j-i+1}{2}, \forall i<j$

We note that if the values of the obtained matrix $\widetilde{P}$ with elements $\tilde{p}_{i j}$ in the interval $[-c, 1+c](c>0)$ not in the interval $[0,1]$. In such a case, the obtained fuzzy numbers would need to be transformed using a transformation function which preserves reciprocity and additive consistency, namely a function $f:[-c, 1+c] \rightarrow[0,1]$, verifying

(1) $f(-c)=0$.

(2) $f(1+c)=1$.

(3) $f\left(x^{L}\right)+f\left(x^{R}\right)=1, \quad \forall x \in[-c, 1+c]$.

(4) $f\left(x^{M}\right)+f\left(x^{M}\right)=1, \quad \forall x \in[-c, 1+c]$.

(5) $f\left(x^{R}\right)+f\left(x^{L}\right)=1, \quad \forall x \in[-c, 1+c]$.

(6)

$f\left(x^{L}\right)+f\left(y^{L}\right)+f\left(z^{R}\right)=\frac{3}{2}, \forall x^{L}, y^{L}, z^{R} \in[-c, 1+c]$ such that $x^{L}+y^{L}+z^{R}=\frac{3}{2}$

(7)

$f\left(x^{M}\right)+f\left(y^{M}\right)+f\left(z^{M}\right)=\frac{3}{2}, \forall x^{M}, y^{M}, z^{M} \in[-c, 1+c]$ such that $x^{M}+y^{M}+z^{M}=\frac{3}{2}$ (8)

$f\left(x^{R}\right)+f\left(y^{R}\right)+f\left(z^{L}\right)=\frac{3}{2}, \forall x^{R}, y^{R}, z^{L} \in[-c, 1+c]$ such that $x^{R}+y^{R}+z^{L}=\frac{3}{2}$

\section{Illustrative example}

To demonstrate the caculation of the proposed method, this study considers CIM systems selection originally presented by Bozdog et al. [3]. A big Turkish Motor Company wants to purchase a CIM system to produce some machinery parts. The top management determined four alternative CIM systems meeting the company's needs. A group of eight evaluators was charged to choose the best CIM system (Goal). The criteria taken into account are better quality (C1), greater flexibility (C2), competition (C3), experience with new technology (C4), and expandability (C5). The alternatives are CIM1 (A), CIM2 (B), CIM3 (C), and CIM4 (D). The hierarchy of the problem is shown in Fig. 1.

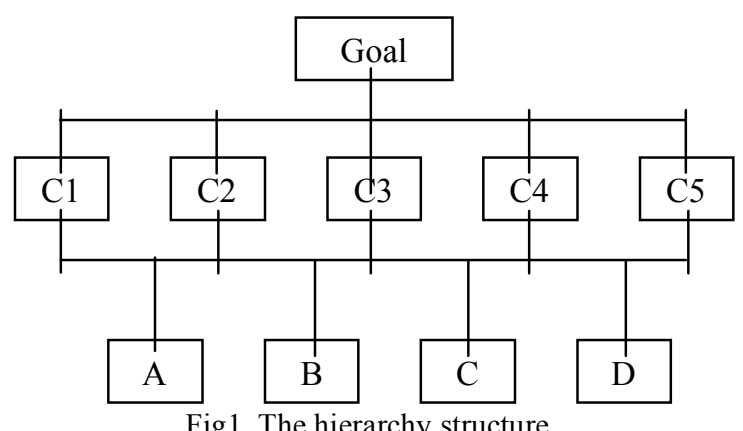

Fig1. The hierarchy structure

Fuzzy linguistic assessment variables are listed in Table 2 by converting Bozdog's Linguistic scale according to $p_{i j}=g\left(a_{i j}\right)=\frac{1}{2} \cdot\left(1+\log _{9.5} a_{i j}\right), p_{i j} \in(0,1), a_{i j} \in(1,9.5)$.

For example,

$(0.1,0.2,0.3)=\left(\frac{1}{2} \cdot\left(1+\log _{9.5} 5 / 2\right), \frac{1}{2} \cdot\left(1+\log _{9.5} 3\right), \frac{1}{2} \cdot\left(1+\log _{9.5} 7 / 2\right)\right)^{.}$

Table 2. Fuzzy linguistic assessment variables

\begin{tabular}{|c|c|c|c|}
\hline $\begin{array}{l}\text { Linguistic } \\
\text { variables }\end{array}$ & $\begin{array}{l}\text { Bozdog's } \\
\text { fuzzy scale }\end{array}$ & $\begin{array}{l}\text { The proposed } \\
\text { method's } \\
\text { fuzzy scale }\end{array}$ & $\begin{array}{l}\text { Reciprocal } \\
\text { scale }\end{array}$ \\
\hline Equally important & $(1,1,1))$ & $(0.5,0.5,0.5)$ & $(0.5,0.5,0.5)$ \\
\hline $\begin{array}{l}\text { Weekly more } \\
\text { important }\end{array}$ & $(5 / 2,3,7 / 2)$ & $(0.1,0.2,0.3)$ & $(0.7,0.8,0.9)$ \\
\hline $\begin{array}{l}\text { Strongly more } \\
\text { important }\end{array}$ & $(9 / 2,5,11 / 2)$ & $(0.2,0.3,0.4)$ & $(0.6,0.7,0.8)$ \\
\hline $\begin{array}{l}\text { Very strongly } \\
\text { more important }\end{array}$ & $(13 / 2$ & $(0.3,0.4,0.5)$ & $(0.5,0.6,0.7)$ \\
\hline $\begin{array}{l}\text { Absolutely more } \\
\text { important }\end{array}$ & $(17 / 2,9,19 / 2)$ & $(0.4,0.5,0.6)$ & $(0.4,0.5,0.6)$ \\
\hline
\end{tabular}

Tables 3 and 4 present the original group pairwise comparisons of all criteria and alternatives. The result of the FAHP method is $\mathrm{A}=0.635, \mathrm{~B}=0.060, \mathrm{C}=0.000$, and $\mathrm{D}=0.305$. The ranks are $\mathrm{A}>\mathrm{D}>\mathrm{B}>\mathrm{C}$.

Table 3. Original pairwise group comparisons of the criteria

\begin{tabular}{llllll}
\hline Goal & $\mathrm{C} 1$ & $\mathrm{C} 2$ & $\mathrm{C} 3$ & $\mathrm{C} 4$ & $\mathrm{C} 5$ \\
\hline $\mathrm{C} 1$ & $(1,1,1)$ & $(3 / 2,2,5 / 2)$ & $(5 / 2,3,7 / 2)$ & $(3 / 2,2,5 / 2)$ & $(11 / 2,6$, \\
$\mathrm{C} 2$ & $(2 / 5,1 / 2,2 / 3)$ & $(1,1,1)$ & $(3 / 2,2,5 / 2)$ & $(1,1,1)$ & $(9 / 2)$ \\
$\mathrm{C} 3$ & $(2 / 7,1 / 3,2 / 5)$ & $(2 / 5,1 / 2,2 / 3)$ & $(1,1,1)$ & $(2 / 5,1 / 2,2 / 3)$ & $(3 / 2,2,5 / 2)$ \\
$\mathrm{C} 4$ & $(2 / 5,1 / 2,2 / 3)$ & $(1,1,1)$ & $(3 / 2,2,5 / 2)$ & $(1,1,1)$ & $(7 / 2,4,9 / 2)$ \\
$\mathrm{C} 5$ & $(2 / 13,1 / 6$, & $(2 / 11,1 / 5$, & $(2 / 5,1 / 2$, & $(2 / 9,1 / 4,2 / 7)$ & $(1,1,1)$ \\
& $2 / 11)$ & $2 / 9)$ & $2 / 3)$ & & \\
\hline
\end{tabular}

Table 4. Original group pairwise comparisons of the alternatives

\begin{tabular}{lllll}
\hline Goal & A & B & C & D \\
\hline C1
\end{tabular}




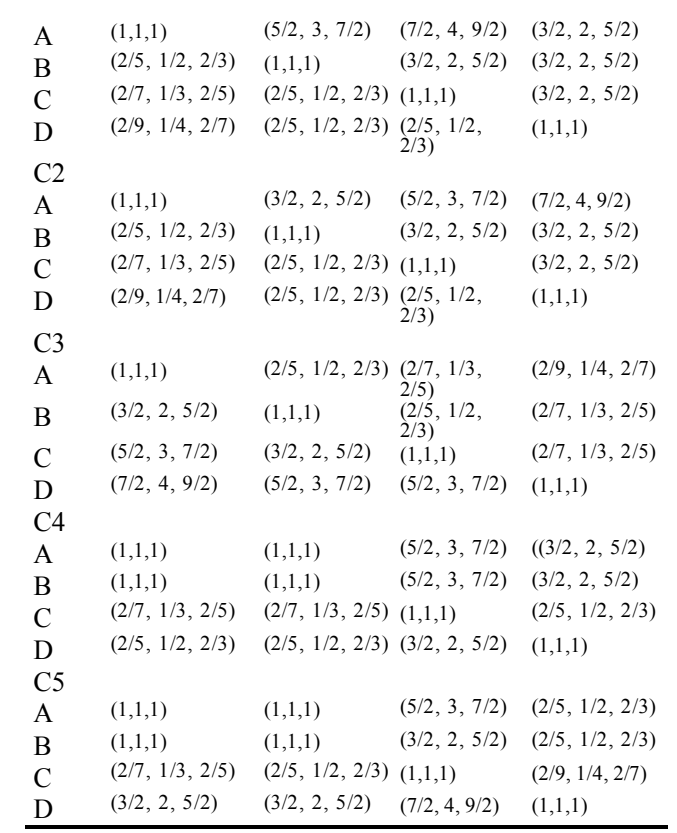

The above example demonstrates how the decision matrices of the proposed method are constructed. The original data in Tables 3 and 4 yield the decision matrices of consistent fuzzy linguistic preference relations according to Propositions 2.1 and 2.2. For instance, Table 3 has five criteria. Only $(n-1=5-1=4) \quad$ values $\left\{p_{12}, p_{23}, p_{34}, p_{45}\right)$ are required to construct the decision matrices. The whole calculation is as follows:

$$
\begin{aligned}
& p_{12}^{L}=\frac{1}{2}\left(1+\log _{9.5} 3 / 2\right)=0.59 \\
& p_{12}^{M}=\frac{1}{2}\left(1+\log _{9.5} 2\right)=0.65 \\
& p_{12}^{R}=\frac{1}{2}\left(1+\log _{9.5} 5 / 2\right)=0.70 \\
& p_{23}^{L}=\frac{1}{2}\left(1+\log _{9.5} 3 / 2\right)=0.59 \\
& p_{23}^{M}=\frac{1}{2}\left(1+\log _{9.5} 2\right)=0.65 \\
& p_{23}^{R}=\frac{1}{2}\left(1+\log _{9.5} 5 / 2\right)=0.70 \\
& p_{34}^{L}=\frac{1}{2}\left(1+\log _{9.5} 2 / 5\right)=0.30 \\
& p_{34}^{M}=\frac{1}{2}\left(1+\log _{9.5} 1 / 2\right)=0.35 \\
& p_{45}^{L}=\frac{1}{2}\left(1+\log _{9.5} 7 / 2\right)=0.78 \\
& p_{34}^{R}\left(1+\log _{9.5} 2 / 3\right)=0.41 \\
& p_{4}=0
\end{aligned}
$$

$$
\begin{aligned}
& p_{45}^{M}=\frac{1}{2}\left(1+\log _{9.5} 4\right)=0.81 \text {, } \\
& p_{45}^{R}=\frac{1}{2}\left(1+\log _{9.5} 9 / 2\right)=0.83 \text {, } \\
& p_{31}^{L}=1.5-p_{12}^{R}-p_{23}^{R}=0.09 \text {, } \\
& p_{31}^{M}=1.5-p_{12}^{M}-p_{23}^{M}=0.19 \text {, } \\
& p_{31}^{R}=1.5-p_{12}^{L}-p_{23}^{L}=0.32 \text {, } \\
& p_{41}^{L}=2-p_{12}^{R}-p_{23}^{R}-p_{34}^{R}=0.18 \text {, } \\
& p_{41}^{M}=2-p_{12}^{M}-p_{23}^{M}-p_{34}^{M}=0.35 \text {, } \\
& p_{41}^{R}=2-p_{12}^{L}-p_{23}^{L}-p_{34}^{L}=0.52 \text {, } \\
& p_{42}^{L}=1.5-p_{23}^{R}-p_{34}^{R}=0.39 \text {, } \\
& p_{42}^{M}=1.5-p_{23}^{M}-p_{34}^{M}=0.50 \text {, } \\
& p_{42}^{R}=1.5-p_{23}^{L}-p_{34}^{L}=0.61 \text {, } \\
& p_{51}^{L}=2.5-p_{12}^{R}-p_{23}^{R}-p_{34}^{R}-p_{45}^{R}=-0.15 \text {, } \\
& p_{51}^{M}=2.5-p_{12}^{M}-p_{23}^{M}-p_{34}^{M}-p_{45}^{M}=0.04 \text {, } \\
& p_{51}^{R}=2.5-p_{12}^{L}-p_{23}^{L}-p_{34}^{L}-p_{45}^{L}=0.25 \text {, } \\
& p_{52}^{L}=2-p_{23}^{R}-p_{34}^{R}-p_{45}^{R}=0.05 \text {, } \\
& p_{52}^{M}=2-p_{23}^{M}-p_{34}^{M}-p_{45}^{M}=0.19 \text {, } \\
& p_{52}^{R}=2-p_{23}^{L}-p_{34}^{L}-p_{45}^{L}=0.34 \text {, } \\
& p_{53}^{L}=1.5-p_{34}^{R}-p_{45}^{R}=0.26 \text {, } \\
& p_{53}^{M}=1.5-p_{34}^{M}-p_{45}^{M}=0.35 \text {, } \\
& p_{53}^{R}=1.5-p_{34}^{L}-p_{45}^{L}=0.43 \text {, } \\
& p_{32}^{L}=1-p_{23}^{R}=0.30, p_{32}^{M}=1-p_{23}^{M}=0.35 \text {, } \\
& p_{32}^{R}=1-p_{23}^{L}=0.41, p_{43}^{L}=1-p_{34}^{R}=0.59 \text {, } \\
& p_{43}^{M}=1-p_{34}^{M}=0.65, p_{43}^{R}=1-p_{34}^{L}=0.70 \text {, } \\
& p_{54}^{L}=1-p_{45}^{R}=0.17, p_{54}^{M}=1-p_{45}^{M}=0.19 \text {, } \\
& p_{54}^{R}=1-p_{45}^{L}=0.22
\end{aligned}
$$

Table 5 lists the consistent fuzzy linguistic preference relation matrix. The matrix has entries that are not contained in the interval $[0,1]$, and thus these transforming functions $f\left(x^{L}\right)=\frac{x^{L}+c}{1+2 c}$, $f\left(x^{M}\right)=\frac{x^{M}+c}{1+2 c}, \quad f\left(x^{R}\right)=\frac{x^{R}+c}{1+2 c}$ are applied. Table 6 lists the Transforming matrix, in which, the weight $W_{i}$ was determined according to $W_{i}=A_{i} / \sum_{i=1}^{n} A_{i}$, 
$A_{i}=\frac{1}{n}\left(\sum_{j=1}^{n} p_{i j}\right)$. Similarly, Table 7 presents the decision matrices of alternatives determined by applying each criterion. Finally, adding the weights per CIM alternative multiplied by the weights of the corresponding criteria, a final score is obtained for each CIM alternative. Table 8 shows these scores. The final score employs the equation $W_{i}=\frac{1}{3}\left(p_{i j}^{L}+p_{i j}^{M}+p_{i j}^{R}\right)$

proposed by Yager [7]. The ranks are A $>$ D $>$ B $>$ C . The result is the same as the original example.

Table 5. Consistent fuzzy linguistic preference relation matrix of criteria

\begin{tabular}{llllll}
\hline Goal & C1 & C2 & C3 & C4 & C5 \\
\hline C1 & $(0.5,0.5,0.5)$ & $(0.59,0.65,0.7)$ & $(0.68,0.81,0.91)$ & $(0.48,0.65,0.82)$ & $(0.75,0.96,1.15)$ \\
C2 & $(0.3,0.35,0.41)$ & $(0.5,0.5,0.5)$ & $(0.59,0.65,0.7))$ & $(0.39,0.5,0.61)$ & $(0.66,0.81,0.95)$ \\
C3 & $(0.09,0.19,0.32)$ & $(0.3,0.35,0.41)$ & $(0.5,0.5,0.5)$ & $(0.3,0.35,0.41)$ & $(0.57,0.65,0.74)$ \\
C4 & $(0.18,0.35,0.52)$ & $(0.39,0.5,0.61)$ & $(0.59,0.65,0.7)$ & $(0.5,0.5,0.5)$ & $(0.78,0.81,0.83)$ \\
C5 & $(-0.15,0.04,0.25)$ & $(0.05,0.19,0.33)$ & $(0.26,0.35,0.43)$ & $(0.17,0.19,0.22)$ & $(0.5,0.5,0.5)$ \\
\hline
\end{tabular}

Table 6. Transforming matrix from Table 5

\begin{tabular}{lllllll}
\hline Goal C1 & C2 & C3 & C4 & C5 & Weight \\
\hline C1 & $(0.5,0.5,0.5)$ & $(0.57,0.62,0.66)$ & $(0.64,0.74,0.81)$ & $(0.48,0.62,0.74)$ & $(0.7,0.86,1)$ & $(0.21,0.27,0.34)$ \\
C2 & $(0.34,0.38,0.43)$ & $(0.5,0.5,0.5)$ & $(0.57,0.62,0.66))$ & $(0.41,0.5,0.59)$ & $(0.63,0.74,0.84)$ & $(0.17,0.22,0.28)$ \\
C3 & $(0.19,0.26,0.36)$ & $(0.34,0.38,0.43)$ & $(0.5,0.5,0.5)$ & $(0.34,0.38,0.43)$ & $(0.56,0.62,0.69)$ & $(0.14,0.17,0.22)$ \\
C4 & $(0.26,0.38,0.52)$ & $(0.41,0.5,0.59)$ & $(0.57,0.62,0.66)$ & $(0.5,0.5,0.5)$ & $(0.71,0.74,0.76)$ & $(0.17,0.22,0.28)$ \\
C5 & $(0,0.14,0.3)$ & $(0.16,0.26,0.39)$ & $(0.31,0.38,0.44)$ & $(0.24,0.26,0.29)$ & $(0.5,0.5,0.5)$ & $(0.09,0.12,0.17)$ \\
\hline
\end{tabular}

Table 7. Weight vectors for the alternatives

\begin{tabular}{cccl}
\hline $\mathrm{A}$ & $\mathrm{B}$ & $\mathrm{D}$ \\
\hline $\mathrm{C} 1(0.28,0.35,0.42)$ & $(0.17,0.2,0.24)$ & $(0.11,0.15,0.19)$ & $(0.25,0.3,0.37)$ \\
$\mathrm{C} 2(0.28,0.37,0.48)$ & $(0.22,0.27,0.34)$ & $(0.17,0.22,0.29))$ & $(0.09,0.14,0.22)$ \\
C3 $(0.12,0.16,0.21)$ & $(0.19,0.22,0.26)$ & $(0.23,0.27,0.31)$ & $(0.3,0.35,0.41)$ \\
C4 $(0.26,0.3,0.34)$ & $(0.24,0.27,0.31)$ & $(0.15,0.18,0.21)$ & $(0.2,0.25,0.31)$ \\
\hline C5 $(0.22,0.25,0.29)$ & $(0.21,0.24,0.26)$ & $(0.15,0.18,0.21)$ & $(0.29,0.33,0.39)$ \\
\hline
\end{tabular}

Table 8. Final scores of the alternatives

\begin{tabular}{lll}
\hline & Fuzzy numbers & Final score \\
\hline A & $(0.19,0.3,0.47)$ & 0.32 \\
B & $(0.16,0.24,0.37)$ & 0.26 \\
C & $(0.12,0.19,0.31)$ & 0.21 \\
D & $(0.17,0.27,0.43)$ & 0.29 \\
\hline
\end{tabular}

\section{Conclusions}

In this study, the consistent fuzzy preference relations are used to derive decision matrices, and the presented method is applied to the example employed by Bozdog (2003). This study reveals that the proposed method yields the same result as that of Bozdog. However, the proposed method can reduce the number of pairwise comparisons. The illustrated example involves five criteria and requires needs four comparisons. There are four alternatives and it only needs three comparisons. Therefore, the number of pairwise comparisons can be reduced by $\left(C_{2}^{5}-4\right)+5 \times\left(C_{2}^{4}-3\right)=21$ and consistency is ensured. The proposed method enhances the efficiency and accuracy of decisions. The increase in the number of criteria and alternatives reduces the number of comparison required by the proposed method. This study indicates that the proposed method simply and practically provides a ranking of alternative solutions to fuzzy decision-making problems

\section{References}

[1] L. A. Zadeh, "Fuzzy Sets", Information and Control, Vol 8, pp. 338-353, 1965.

[2] P. J. M. Laarhoven and W. Pedrycz, "A Fuzzy Extension of Saaty's Priority Theory”, Fuzzy Sets and Systems, Vol 11, pp. 229-241, 1983.

[3] C. E. Bozdag, C. Kahraman and D. Ruan, "Fuzzy group decision making for selection among computer integrated manufacturing systems", Computers in Industry, Vol 51, pp. 1329, 2003.

[4] T. C. Wang and Y. H. Chen, "A New Method on Decision-Making Using Fuzzy Linguistic Assessment Variables and Fuzzy Preference Relations", The Proceedings of the $9^{\text {th }}$ World Multi-Conference on Systemics, Cybernetics and Informatics, Orlando, pp. 360-363, July 2005.

[5] E. Herrera-Viedma, F. Herrera, F. Chiclana and M. Luque, "Some Issues on Consistency of Fuzzy Preference Relations", European Journal of Operational Research, Vol 154, pp. 98-109, 2004.

[6] J. J. Buckley, "Fuzzy Hierarchical Analysis", Fuzzy Sets and Systems, Vol 17, pp. 233-247, 1985.

[7] R. R. Yager, "On a General Class of Fuzzy Connectives", Fuzzy Sets and Systems, Vol 14, 1981. 